\title{
Revisión sistemática: los medicamentos para la tos no son efectivos en los cuadros agudos
}

Should we advise parents to administer over the counter cough medicines for acute cough?.Systematic review of randomised controlled trials. Schroeder K., Fahey T. Arch Dis Child 2002; 86:170-175.

\section{Objetivo}

Evaluar la eficacia de los medicamentos para la tos de venta libre, en chicos con cuadros tusígenos agudos en infecciones del tracto respiratorio superior.

Diseño

Revisión sistemática

\section{Origen de los datos}

Se evaluaron los registros especializados del Grupo de Infecciones respiratorias Agudas Cochrane, Registro de Ensayos Controlados Cochrane, Medline y Embase (ambos hasta Diciembre 1999), y los registros del departamento Nacional de Investigación de la Salud de Gran Bretaña (Diciembre 2000).

\section{Estudios seleccionados}

Ensayos clínicos aleatorizados controlados (ECCA). Criterios de inclusión:

-Chicos (menores de 16 años) con cuadro agudo de tos (menor de tres semanas), resultante de una infección del tracto respiratorio superior (ITRS) y tratados ambulatoriamente.

-Que la intervención sea la de preparados para la tos de venta libre. -Que los resultados reportados fueran relacionados con la tos $(\mathrm{Ej}$. frecuencia, duración)

-Que los grupos de medicamento activo sean compárados con grupos de control con placebo.

Fueron excluidos:

- Cuadros de tos crónica (más de tres semanas o causada por enfermedades crónicas subyacentes, como asma)

- Cuadros de tos provocada artificialmente en voluntarios sanos.

-Tratamientos no convencionales (ej: homeopatía) y por vía no oral.

\section{Resultados principales}

Solo seis ensayos (total: 438 chicos), reunieron la mayoría de los criterios de inclusión; dividiéndose en relación con cinco grupos de drogas: (A) antitusivos, (B) mucolíticos, (C) antihistamínicos más descongestivos, (D) otras combinaciones y (E) antihistamínicos solos.
La calidad metodológica de los estudios fue en general baja.

-(A) Un estudio evaluó dextrometorfano y codeína vs. placebo,dando una sola dosis nocturna durante tres días. Ninguna de las drogas fue más efectiva que el placebo al tercer día. Los efectos adversos (adormecimiento, diarrea e hiperactividad) no tuvieron diferencias estadísticamente significativas entre los grupos.

-(B) Un estudio comparó Letosterina vs. placebo. Mejores resultados se apreciaron con Letosterina entre los días cuatro y diez (diferencia de poca significancia clínica, aunque sí estadística). Sin reportarse efectos adversos.

-(C) Se incluyeron dos estudios: uno que comparó una combinación de brompeniramina/ fenilpropanolamina no siendo más efectiva que el placebo en reducir el número de tosedores a las dos horas de administrada cada dosis (49.0\% vs. $43.1 \%$ p: 0.66$)$, reportándose mas chicos con adormecimiento en el grupo de intervención vs. el placebo ( $46.6 \%$ vs. $26.5 \%$ p: 0.53). El otro evaluó bromperinamina/ fenilefrina/ fenilpropanolamina, no siendo más efectivos que el placebo ( $67 \%$ vs. $58 \%$ y $70 \%$ p: 0.5 y p: 0.8 respectivamente)

-(D) Se evaluó un estudio que incluía almíbares pediátricos para la tos (Triaminicol y Dorcol). El $69 \%$ de los chicos de ambos grupos mostraron respuestas satisfactorias comparados con el $57 \%$ de los del grupo placebo (p: 0.5). No se reportaron efectos adversos.

(E) Incluyó un ensayo que comparó al Clemastine y la clorfeniramina con placebo. Los que recibieron tratamiento activo presentaron mejoría de la tos en un $39.6 \%$ vs. un $27.6 \%$ del grupo placebo (p: 0.2 ). Se reportó adormecimiento y sueño en un $20 \%$, no existiendo diferencias estadísticamente significativas entre los grupos.

\section{Conclusión}

Los medicamentos utilizados para resolver los cuadros agudos de tos, causados por ITRS, no parecen ser más efectivos que el placebo en resolver este síntoma. No deben recomendarse como primera línea de tratamiento en estos casos; aunque sean generalmente bien tolerados.

\section{Comentario}

La TOS es un síntoma que preocupa a la mayoría de los padres, ya sea por el "que tendrá", o por el "no está descansando" y en algunos casos por el "no deja descansar". Todas estas apreciaciones hacen que en muchos casos recurran a la compra (con o sin indicación médica) de los comúnmente llamados "jarabes para la tos": sin conocer su real efectividad, costos y efectos adversos. Este trabajo cuenta con un reducido número de ensayos, lo cual está íntimamente relacionado con la escasa disponibilidad de datos. Los estudios fueron muy heterogéneos, con amplias diferencias entre ellos tanto en lo que hace al tipo de intervención y medición de resultados como a la calidad metodológica de los mismos Se pudo comprobar que existe poca evidencia para avalar la efectividad de los medicamentos de venta libre utilizados en los cuadros aqudos de tos; y tampoco se hallaron diferencias estadísticamente significativas al compararlos con placebo. Esto resalta el concepto de que la tos aguda y limitada no requiere tratamiento, siendo la prioridad inicial la identificación de los factores desencadenantes y su etiología

Diferentes trabajos evaluaron el uso de brompeniramina y clemastine (entre otros) para el tratamiento de los síntomas que acompañan a la tos (rinorea, estornudo, etc.) en las ITRS ${ }^{2,3,4}$. Sin embargo muy pocos evaluaron sus efectos sobre la tos.

Conclusiones del Comentador: Sobre la base de la evidencia disponible hasta el momento, podemos concluir que estos medicamentos no deben ser indicados como primera línea de tratamiento para los cuadros agudos de tos causados por ITRS, aunque su tolerancia sea buena.

Es indispensable destinar un tiempo prudencial de la consulta a la "consejería", para reafirmar estos conceptos en los padres.

\section{Dr. Javier Chiarpenello [ Médico Jefe de Centro de Salud № 29. Atención Primaria de Salud . Hospital Provincial de Rosario. ]}

\section{Referencias}

- The Diagnosis and Treatment of Cough. Irwin,RS; Madison,JM. N.Engl.J.Med.2000;343:1715

- Randomized controlled trial of clemastine fumarate for treatment of experimental thinovirus colds. Gwaltney, JM Jr, Park, J; Paul, RA; et al. Clin. Infect. Dis. 1996;22:656.

Effectiveness of clemastine fumarato for treatment of rhinorrhea and sneezing associated with the common cold.Turner,RB; Sperber,SJ; Soneutino, JV, et al. Clin. Infect. Dis. 1997;25:824.

4. Efficacy of brompheniramine maleate for the treatment of rhinovirus colds. Qwaltney,JM Jr; Druce, HM. Clin. Infect. Dis. 1997;25:1188. 